\title{
Effects of a transmitted light device for pediatric peripheral venipuncture and intravenous cannulation
}

This article was published in the following Dove Press journal:

Medical Devices: Evidence and Research

30 September 2011

Number of times this article has been viewed

\author{
Shinya Yamazaki' \\ Shu Tomita' \\ Masahiro Watanabe' \\ Hiroyoshi Kawaai' \\ Kazuhiro Shimamura ${ }^{2}$ \\ 'Department of Dental \\ Anesthesiology; ${ }^{2}$ Department of \\ Pediatric Dentistry, Ohu University \\ Dental Hospital, Koriyama City, \\ Fukushima Prefecture, Japan
}

\begin{abstract}
Pediatric peripheral venipuncture and intravenous cannulation are difficult. However, successful venipuncture and intravenous cannulation are absolutely required for pediatric clinical risk management. This study assessed the success rate of venipuncture and intravenous cannulation when transmitted light was applied to the pediatric dorsum manus. The subjects included 100 young children who were scheduled for dental treatment or oral surgery under general anesthesia. Anesthesia was induced, and insertion of an intravenous catheter into the dorsum manus was attempted with or without using transmitted light. The patients were evaluated to determine whether the venipuncture was successful, and whether the intravenous cannulation of the external catheter was successful. The success rate of venipuncture was $100 \%$ when transmitted light was used, and $83 \%$ when the transmitted light was not used $(P=0.000016)$. In addition, the success rate of intravenous cannulation was $88 \%$ when transmitted light was used, and 55\% when the transmitted light was not used $(P=0.0000002)$. The shape of the vein in the dorsum manus can be clearly recognized when transmitted light is used. The use of light significantly increased the success rate of intravenous cannulation, because it allowed direct confirmation of the direction to push the intravenous catheter forward. The use of transmitted light allows for more successful venipuncture and intravenous cannulation in young children.

Keywords: transmitted light, pediatric peripheral venipuncture, pediatric peripheral intravenous cannulation
\end{abstract}

\section{Introduction}

Pediatric peripheral venipuncture (PPV) and pediatric peripheral intravenous cannulation (PPIVC) are difficult even for skilled practitioners. ${ }^{1,2}$ The average success rate for PPIVC was only 592/1135 (52\%) even for two large institutions in the United States, ${ }^{1}$ although the success rate correlates with the competence or experience of the inserter up to a point. In our institution, the average success rate for PPIVC was almost the same as in the above report. ${ }^{1}$ It is speculated that many institutions have the same problems with low success rates in PPV and PPIVC. Therefore, successful or foolproof strategies for PPV and PPIVC are urgently needed for clinical risk management.

There was one previous report about the use of transmitted light for PPV or PPIVC, but the report just introduced the method and did not include any clinical data. $^{3}$ Therefore, there have been no reports which have included clear data showing the impact of using transmitted light on the success rate of PPV or PPIVC. Although there have been reports of using an ultrasound device ${ }^{4}$ and a near-infrared device ${ }^{5}$ for PPV or PPIVC as alternatives to transmitted light, it is still difficult to visualize the peripheral veins or the deeper veins, even when using these expensive instruments.
Correspondence: Shinya Yamazaki Department of Dental Anesthesiology, Ohu University Dental Hospital, 3I-I Misumido, Tomita Koriyama, Fukushima, 963-86II Japan

Tel +8I 249329337

Fax +8I 249389192

Email zakiyama@ops.dti.ne.jp 
Therefore, this study assessed the success rates of PPV and PPIVC when transmitted light was applied to the pediatric dorsum manus.

\section{Subjects and methods}

Our hospital accepts a lot of pediatric ambulatory general anesthesia cases from private dental clinics, including those who require anesthesia for strong treatment refusal or because of severe dental phobia. These children usually require the insertion of an intravenous line immediately after the induction of anesthesia for intravenous fluid infusion and/or for sampling for immediate blood examination. In the present study, after induction of anesthesia, insertion of an intravenous catheter (Insyte ${ }^{\mathrm{TM}} 24$ gauge, Becton Dickinson, Franklin Lakes, NJ) into the patient's dorsum manus was attempted with or without using transmitted light (Figure 1). Fiberoptic light (558B, Karl Storz, Tuttlingen, Germany) was used as a source of transmitted light, because the fiber tip does not become hot in the presence of strong light. The subjects included 100 healthy children who were scheduled to undergo a dental procedure under general anesthesia. In order for the statistical type II error to be less than $20 \%$, the necessary sample size was calculated to be 100 . This study was designed as a randomized controlled trial whereby the subjects were randomized into two groups, ie, those with the use of transmitted light (50 subjects) versus those without the use of transmitted light (50 subjects), and informed consent was obtained for this institutional review boardapproved study.

Of the 10 anesthetists in our department, the newest/least experienced anesthetists and the most senior anesthetists were excluded as inserters in this study, because the success rates of PPV and PPIVC should be adjusted to the average level. Therefore, five anesthetists (training period 3-5 years) were chosen to perform the PPV and PPIVC. Their usual success rates for PPIVC were approximately the same as the average level indicated in a previous report $(52 \%){ }^{1}$

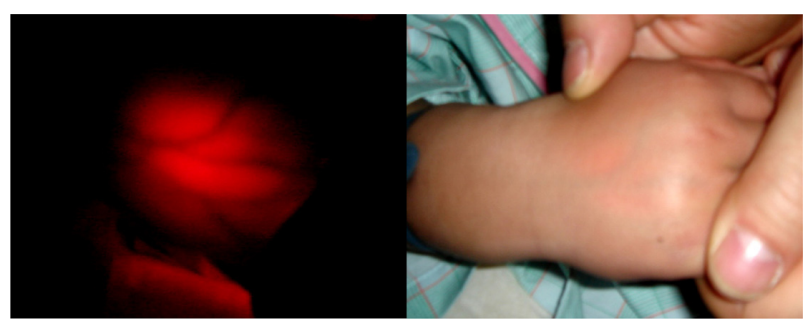

Figure I Pediatric peripheral venipuncture and intravenous cannulation into the dorsum manus were attempted with or without the use of transmitted light. The shape of the vein in the dorsum manus can be clearly recognized by the transmitted light.
Each inserter performed PPV and PPIVC for 20 hands of 20 subjects. While the inserter performed the PPV and PPIVC under transmitted light, an assistant nurse supported the patient's dorsum manus from the palm side to ensure that it was illuminated. The operating room lights were dimmed appropriately when the transmitted light was used. The inserters did not have any previous experience with using transmitted light for PPV and PPIVC. If blood backflow was not recognized within one minute from the start of insertion into the dorsal manus skin, it was defined as failure of the PPV. If the intravenous catheter was not placed in the vein within 2 minutes from the start of insertion into the dorsal manus skin, it was defined as failure of PPIVC. The assistant nurse, as an objective third party, evaluated the procedures for success versus failure. If the first attempt for one patient was a failure, a fully trained senior anesthetist performed the second attempt to ensure ethical treatment of the patient. This study could not be designed as a double-blind study, because the inserter would notice whether the light was being used. However, the inserter and the subject's hand (right/left) were chosen randomly by an objective third party.

\section{Statistical analysis}

A $2 \times 2$ Chi-square test was used to for the statistical analysis, and $P<0.05$ was considered to be significant.

\section{Results}

The demographic and clinical characteristics of the 100 subjects are shown in Table 1 . The success rate of PPV was $100 \%$ when transmitted light was used, and $83 \%$ when the transmitted light was not used. There was a significant difference $(P=0.000016)$ between the two conditions (Figure 2). In addition, the success rate of PPIVC was $88 \%$ when transmitted light was used, and 55\% when the transmitted light was not used. There was a significant difference $(P=0.0000002)$ between the two conditions (Figure 2).

Table I Clinical and demographic characteristics of subjects

\begin{tabular}{ll}
\hline Number (cases) & 100 \\
Gender (Male:female) & $59: 41$ \\
Age (years) & $3.8 \pm 1.6$ \\
Height (cm) & $92 \pm 14$ \\
Weight (kg) & $14 \pm 7$ \\
ASA-PS & 1 or 2 \\
Main diagnosis for general anesthesia (cases) \\
$\quad$ Dental caries & 76 \\
$\quad$ Impacted teeth & 12 \\
$\quad$ Cleft palate & 9 \\
$\quad$ Tumor & 4 \\
\hline Abbreviation: ASA-PS, American Society of Anesthesiologists-Physical Status.
\end{tabular}



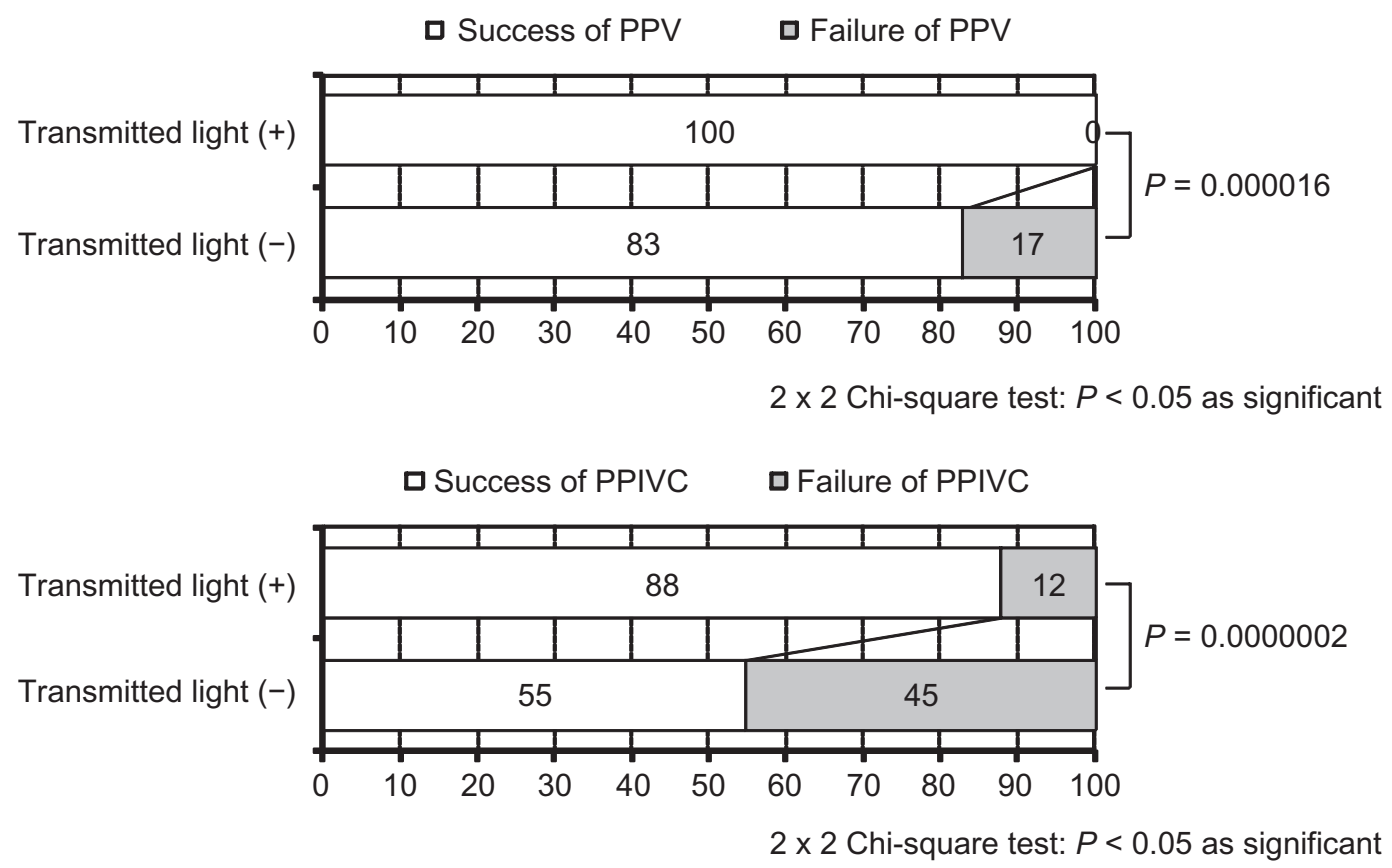

Figure 2 Success rate of PPV and PPIVC.

Abbreviations: PPV, pediatric peripheral venipuncture; PPIVC, pediatric peripheral intravenous cannulation.

\section{Discussion}

There has been one previous report about the use of transmitted light for PPV or PPIVC, but the report just introduced the method and did not include any clinical data. ${ }^{3}$ Although there are some medical organizations which apply transmitted light for difficult venipuncture of the pediatric dorsum manus, there have been no reports that have investigated the effect of transmitted light for clinical use. Shimawaki et al used transmitted light clinically for obtaining images of blood vessels to speculate the vein compliance in the subject's finger while the upper arm of the subject was compressed. ${ }^{6}$ However, most of the relevant research about transmitted light in the human hand is for personal identification using vein patterns, and not for clinical use. ${ }^{7}$

There are some medical organizations which have adopted the use of ultrasound devices for PPV and PPIVC. ${ }^{4}$ These devices are effective for the visualization of larger vessels, such as the jugular vein, subclavian vein, or femoral vein. ${ }^{8}$ However, the device still does not provide adequate visualization of the peripheral veins, such as those of the dorsum manus, because the image is not clear even when using such an expensive instrument. In addition, there are some medical organizations which have adopted the use of a near-infrared device for PPV and PPIVC. ${ }^{5}$ The device is effective for the visualization of superficial veins. However, it is still difficult to visualize the deeper veins using this instrument, which is also quite expensive.
The shape of the vein in the dorsum manus can be clearly recognized under transmitted light. Use of light in our study significantly increased the success rate of PPIVC, because it makes it possible to confirm directly the direction in which to push the intravenous catheter forward. In particular, PPV was successful in $100 \%$ of the cases when the transmitted light was used.

Some commercial transmitted light systems using lightemitting diodes operated by dry cell batteries are available for clinical use. The price of such a light source is approximately $\$ 100$. However, the battery-operated light-emitting diodes are too weak to demonstrate clearly the shape of the deep veins. Therefore, a strong fiberoptic light was used in this study, because it provides a more powerful light source and has an unheated emission tip. It is necessary to use a light that does not produce significant heat, because a hot light can burn the patient's hand. The Storz fiberoptic light system used in this study is an illumination system commonly used for the surgical field, and is installed on a band situated on the surgeon's forehead. This system provides a strong light, and the majority of institutions have such a system. Ordinary fiberoptic head lights with a cold light source are sold for approximately $\$ 4000$. No complications, such as burns, were observed in this study. Although we found the fiberoptic system to be highly effective, any system that can provide a strong light source and has an unheated emission tip could be used to transmit light for PPV or PPIVC. 
However, the marketing of a purpose-built product that provides a strong light and has an unheated emission tip will eventually be required. In addition, the blood backflow area should be illuminated with a small light, because blood backflow is not easily confirmed when the surroundings are dark. The introduction of this technology should improve the operating room workflow and the clinical risk management, because it will minimize the time, number of trials, and patient injuries associated with PPV and PPIVC.

\section{Conclusion}

The use of transmitted light is effective for performing PPV and PPIVC because it significantly increases the success rate.

\section{Disclosure}

The authors report no conflicts of interest in this work.

\section{References}

1. Larsen P, Eldridge D, Brinkley J, et al. Pediatric peripheral intravenous access: does nursing experience and competence really make a difference? J Infus Nurs. 2010;33:226-235.

2. Eldridge DL. Alternatives to intravenous rehydration in dehydrated pediatric patients with difficult venous access. Pediatr Emerg Care. 2010;26:529-535.

3. Sieh A, Brentin L. A little light makes venipuncture easier. $R N$. 1993;56: $40-43$.

4. Bair AE, Rose JS, Vance CW, Andrada-Brown E, Kuppermann N. Ultrasound-assisted peripheral venous access in young children: a randomized controlled trial and pilot feasibility study. West J Emerg Med. 2008;9:219-224.

5. Perry AM, Caviness AC, Hsu DC. Efficacy of a near-infrared light device in pediatric intravenous cannulation: a randomized controlled trial. Pediatr Emerg Care. 2011;27:5-10.

6. Shimawaki S, Sasaki N, Tamura H. Change in blood vessel image in human finger using near-infrared radiation while compressing the upper arm. The Japan Society of Mechanical Engineers. 2009;75: $2280-2285$.

7. Kono M, Ueki H, Umemura S. Near-infrared finger vein patterns for personal identification. Appl Opt. 2002;41:7429-7236.

8. Faraoni D. Routine use of ultrasound to guide internal jugular vein access in children. Paediatr Anaesth. 2010;20:777-778.
Medical Devices: Evidence and Research

\section{Publish your work in this journal}

Medical Devices: Evidence and Research is an international, peerreviewed, open access journal that focuses on the evidence, technology, research, and expert opinion supporting the use and application of medical devices in the diagnosis, treatment and management of clinical conditions and physiological processes. The identification of novel

\section{Dovepress}

devices and optimal use of existing devices which will lead to improved clinical outcomes and more effective patient management and safety is a key feature. The manuscript management system is completely online and includes a quick and fair peer-review system. Visit http://www. dovepress.com/testimonials.php to read real quotes from authors. 